Discussiones Mathematicae

Differential Inclusions, Control and Optimization 33 (2013) 5-16

doi:10.7151/dmdico.1143

\title{
INPUT-TO-STATE STABILITY OF NEUTRAL TYPE SYSTEMS
}

\author{
MiChAEL I. GiL' \\ Department of Mathematics \\ Ben Gurion University of the Negev \\ P.O. Box 653, Beer-Sheva 84105, Israel \\ e-mail: gilmi@bezeqint.net
}

\begin{abstract}
We consider the system

$$
\dot{x}(t)-\int_{0}^{\eta} d \tilde{R}(\tau) \dot{x}(t-\tau)=\int_{0}^{\eta} d R(\tau) x(t-\tau)+[F x](t)+u(t)
$$

$\left(\dot{x}(t) \equiv \frac{d x(t)}{d t}\right)$, where $x(t)$ is the state, $u(t)$ is the input, $R(\tau), \tilde{R}(\tau)$ are matrix-valued functions, and $F$ is a causal (Volterra) mapping. Such equations enable us to consider various classes of systems from the unified point of view. Explicit input-to-state stability conditions in terms of the $L^{2}$-norm are derived. Our main tool is the norm estimates for the matrix resolvents, as well as estimates for fundamental solutions of the linear parts of the considered systems, and the Ostrowski inequality for determinants.
\end{abstract}

Keywords: neutral type systems, causal mappings, input-to-state stability.

2010 Mathematics Subject Classification: 93D25, 34K40.

\section{INTRODUCTION AND DEFINITIONS}

The present paper is devoted to vector equations with linear functional-differential parts and nonlinear causal mappings (operators). These equations include various traditional differential, functional differential and integro-differential equations. For the details see the excellent book [2]. So the equations with causal mappings enable us to consider various classes of systems from the unified point of view.

The stability theory of nonlinear equations with causal mappings is at an early stage of development. The basic method for the stability analysis is the direct Liapunov method. But finding the Liapunov functionals for equations with 
causal mappings is a difficult mathematical problem. Below we establish explicit conditions that provide the input-to-state stability for the considered systems. The literature on the input-to-state stability of continuous systems is rather rich, cf. $[1,15,20]$ and references therein. The input-to-state and input-output stability of nonlinear retarded systems with causal mappings was investigated considerably less than the one for systems without delay. In papers [6] and [8], bounded input-to-bounded output stability conditions for multivariable retarded systems was derived via the Karlson inequality. In the paper [9], the author has derived a criterion for the $L^{2}$-input-to-state stability of one-contour retarded systems with causal mappings, that is, for systems governed by scalar functional differential equations. At the same time, to the best of our knowledge the input-to-state stability of nonlinear neutral type delay systems especially with causal mappings was not investigated in the available literature. In this paper we improve and generalize the main result from [9].

The paper is organized as follows. It consists of 6 sections. In this section we present some definitions. In Section 2, we prove the existence results and estimates for solutions. The main result is formulated in Section 3. Auxiliary results on scalar equations are established in Section 4. In Section 5 we explore systems with discrete delays. The illustrative example is presented in Section 6 .

By $\mathbb{C}^{n}$ we denote a Euclidean space with the Euclidean norm $\|\cdot\|_{n}$ and the unit matrix $I, L^{2}(a, b)=L^{2}\left([a, b], \mathbb{C}^{n}\right)$ is the spaces of functions $w$ defined on a real segment $[a, b]$ with values in $\mathbb{C}^{n}$ and equipped with the norms

$$
\|w\|_{L^{2}(a, b)}=\left[\int_{a}^{b}\|w(t)\|_{n}^{2} d t\right]^{1 / 2} .
$$

For a linear operator $A$ in $\mathbb{C}^{n}$ (matrix), $\lambda_{k}=\lambda_{k}(A)(k=1, \ldots, n)$ are the eigenvalues of $A$ numerated in an arbitrary order with their multiplicities, $\|A\|_{n}=$ $\sup _{x \in \mathbb{C}^{n}}\|A x\|_{n} /\|x\|_{n}$ is the spectral norm, $N_{2}(A)$ is the Hilbert-Schmidt (Frobenius) norm of $A$ : $N_{2}^{2}(A)=$ Trace $A A^{*}$.

For a positive $\eta<\infty$, and an input $u \in L^{2}(0, \infty)$, consider in $\mathbb{C}^{n}$ the problem

$$
\begin{gathered}
\dot{x}(t)-\int_{0}^{\eta} d \tilde{R}(\tau) \dot{x}(t-\tau)=\int_{0}^{\eta} d R(\tau) x(t-\tau)+[F(x)](t)+u(t), \\
x(t)=0 \text { for }-\eta \leq t \leq 0,
\end{gathered}
$$

where $x(t)$ is the state, $\dot{x}(t) \equiv \frac{d x(t)}{d t}, R(s)=\left(r_{i j}(s)\right)_{i, j=1}^{n}$ and $\tilde{R}(s)=\left(\tilde{r}_{i j}(s)\right)_{i, j=1}^{n}$ are real $n \times n$-matrix-valued functions defined on $[0, \eta]$, whose entries have bounded variations $\operatorname{var}\left(r_{i j}\right)$ and $\operatorname{var}\left(\tilde{r}_{i j}\right)$. The integrals in (1.1) are understood in the sense of the Lebesgue-Stieltjes integral, cf. [14]. In addition, $u \in L^{2}(0, \infty)$ and $F$ is $a$ causal (Volterra) mapping acting in $L^{2}(-\eta, \infty)$ (see Definition below). 
We define the variation of $R($.$) as the matrix \operatorname{Var}(R)=\left(\operatorname{var}\left(r_{i j}\right)\right)_{i, j=1}^{n}$ and denote $\operatorname{var}(R):=\|\operatorname{Var}(R)\|_{n}$. So $\operatorname{var}(R)$ is the spectral norm of matrix $\operatorname{Var}(R)$. Similarly $\operatorname{var}(\tilde{R})$ is defined. It is assumed that

$$
\operatorname{var}(\tilde{R})<1
$$

Let us explain the definition of a causal mapping. For a positive $T \leq \infty$, let $X(\eta, T)$ be a space of vector-valued functions defined on $[-\eta, T]$. For example, $X(-\eta, T)=L^{2}(-\eta, T)$. For all $\tau \in(-\eta, T)$, let $P_{\tau}$ be the projections defined by

$$
\begin{gathered}
\left(P_{\tau} w\right)(t)= \begin{cases}w(t) & \text { if }-\eta \leq t \leq \tau, \\
0 & \text { if } \tau<t \leq T,\end{cases} \\
P_{-\eta} w=0 ; P_{T} w=w(w \in X(-\eta, T)) .
\end{gathered}
$$

Definition. A mapping $\hat{F}: X(-\eta, T) \rightarrow X(-\eta, T)$, satisfying the conditions $\hat{F} 0 \equiv 0$ and

$$
P_{\tau} \hat{F} P_{\tau}=P_{\tau} \hat{F} \quad(\tau \in[-\eta, T])
$$

will be called a causal mapping (operator).

This definition is somewhat different from the definition of the causal operator suggested in [2]. In the linear case our definition is similar to the one accepted in [3]. About various examples of causal operators see for instance, [2]. See also $[8,9]$ and Section 6 below.

\section{Preliminaries}

Let $F$ be a continuous causal mapping in $L^{2}(-\eta, \infty)$ satisfying the following condition: there is a constant $q$, such that

$$
\|F w\|_{L^{2}(0, \infty)} \leq q\|w\|_{L^{2}(-\eta, \infty)}\left(w \in L^{2}(-\eta, \infty)\right) .
$$

A (mild) solution of problem (1.1), (1.2) is a continuous function $x(t)$ defined on $[0, \infty)$, such that

$$
x(t)=\int_{0}^{t} G\left(t-t_{1}\right)\left([F x]\left(t_{1}\right)+u\left(t_{1}\right)\right) d t_{1}(t \geq 0),
$$

$$
x(t)=0(t<0),
$$


where $G(t)$ is the fundamental solution of the linear equation

$$
\dot{x}(t)-\int_{0}^{\eta} d \tilde{R}(s) \dot{x}(t-s)-\int_{0}^{\eta} d R(s) x(t-s)=0(t \geq 0) .
$$

Use the operator $\hat{G}$ defined on $L^{2}(0, \infty)$ by

$$
\hat{G} f(t)=\int_{0}^{t} G\left(t-t_{1}\right) f\left(t_{1}\right) d t_{1} \quad\left(f \in L^{2}(0, \infty)\right),
$$

and assume that

$$
\|\hat{G}\|_{L^{2}(0, \infty)}<\frac{1}{q} .
$$

Lemma 1. Let conditions (1.3), (2.1) and (2.5) hold. Then problem (1.1), (1.2) has a unique solution $x(t)$. Moreover

$$
\|x\|_{L^{2}(0, \infty)} \leq \frac{\|\hat{G}\|_{L^{2}(0, \infty)}\|u\|_{L^{2}(0, \infty)}}{1-q\|\hat{G}\|_{L^{2}(0, \infty)}} .
$$

Proof. Take a finite $T>0$ and define the mapping $\Phi$ by

$$
\Phi w(t)=\int_{0}^{t} G\left(t-t_{1}\right)\left([F x]\left(t_{1}\right)+u\left(t_{1}\right)\right) d t_{1} \quad\left(0 \leq t \leq T ; w \in L^{2}(0, T)\right),
$$

and $\Phi w(t)=0$ for $-\eta \leq t \leq 0$. Then by (2.1) and (1.4) we have

$$
\|\Phi w\|_{L^{2}(-\eta, T)} \leq\|\hat{G}\|_{L^{2}(0, \infty)}\left(q\|w\|_{L^{2}(-\eta, T)}+l\right),
$$

where

$$
l=\|u\|_{L^{2}(0, \infty)} .
$$

So $\Phi$ maps $L^{2}(-\eta, T)$ into itself. Taking into account that $\Phi$ is compact we prove the existence of solutions. Furthermore,

$$
\|x\|_{L^{2}(0, T)}=\|\Phi x\|_{L^{2}(0, T)} \leq\|\hat{G}\|_{L^{2}(0, T)}\left(q\|x\|_{L^{2}(0, T)}+l\right) .
$$

Hence we obtain (2.5), which provides the uniqueness, as claimed.

The matrix-valued function

$$
K(z)=I z-z \int_{0}^{\eta} \exp (-z s) d \tilde{R}(s)-\int_{0}^{\eta} \exp (-z s) d R(s)(z \in \mathbb{C})
$$


is the characteristic matrix-valued function to equation (2.4) and the zeros of det $K(\lambda)$ are the characteristic values of $K(.) ; \lambda \in \mathbb{C}$ is a regular value of $K($. if $\operatorname{det} K(\lambda) \neq 0$. Due to Theorem 3.1.1 from [14, p. 114], under conditions (1.3) equation (1.1) is asymptotically stable and $L^{2}$-stable, if all the characteristic values of $K($.$) are in the open left half-plane C_{-}$. So

$$
G(t):=\frac{1}{2 \pi} \int_{-\infty}^{\infty} e^{-i s t} K^{-1}(i s) d s
$$

As it is well-known, $G(t)$ is a solution to (2.4) with the conditions

$$
G(t)=0(t<0) \text { and } G(0)=I .
$$

Furthermore, put

$$
v_{0}=\frac{2 \operatorname{var}(R)}{1-\operatorname{var}(\tilde{R})} \text { and } \theta(K):=\sup _{-v_{0} \leq \omega \leq v_{0}}\left\|K^{-1}(i \omega)\right\|_{n} .
$$

Lemma 2. The equality $\sup _{-\infty \leq \omega \leq \infty}\left\|K^{-1}(i \omega)\right\|_{n}=\theta(K)$ is valid.

For the proof see [12, Lemma 2].

By the Parseval equality and Lemma 2 we have $\|\hat{G}\|_{L^{2}(0, \infty)}=\theta(K)$. Now Lemma 1 implies

Corollary 3. Let conditions (1.3) and (2.1) hold, and all the zeros of $K$ be in $C_{-}$. If, in addition, $q \theta(K)<1$, then (1.1) has at least one solution. Moreover, any solution $x(t)$ of problem (1.1), (1.2) satisfies the inequality

$$
\|x\|_{L^{2}(0, \infty)} \leq(1-q \theta(K))^{-1} \theta(K) l .
$$

About other existence results for equations with causal mappings see [10]. Clearly, Corollary 3 gives us the global stability condition for neutral type systems with causal nonlinearities. Note that the interesting conditions for the global asymptotic stability for nonlinear neutral-type systems governed neural networks with distributed time delays were suggested in [19]. In that paper the nonlinearity is a vector-valued function continuously mapping $\mathbb{R}^{n}$ into itself.

\section{The MAIN RESUlT}

We will say that system (1.1) is input-to-state $L^{2}$-stable, if for any $\epsilon>0$, there is a $\delta>0$, such that $\|u\|_{L^{2}(0, \infty)} \leq \delta$ implies $\|x\|_{L^{2}\left(R_{+}\right)} \leq \epsilon$ for any solution of problem (1.1), (1.2). 
Furthermore, from [7, Theorem 2.11] it follows that

$$
\left\|A^{-1} \operatorname{det}(A)\right\|_{n} \leq \frac{N_{2}^{n-1}(A)}{(n-1)^{(n-1) / 2}}
$$

for any invertible $n \times n$-matrix $A$. Hence, for any regular point $z$ of $K($.$) , one has$

$$
\left\|K^{-1}(z)\right\|_{n} \leq \frac{N_{2}^{n-1}(K(z))}{(n-1)^{(n-1) / 2}|\operatorname{det}(K(z))|},
$$

and thus

$$
\theta(K) \leq \theta_{d}(K):=\sup _{|\omega| \leq v_{0}} \frac{N_{2}^{n-1}(K(i \omega))}{(n-1)^{(n-1) / 2}|\operatorname{det}(K(i \omega))|}
$$

Now Corollary 3 implies our main result.

Theorem 4. Let the conditions (1.3), (2.1) and $q \theta_{d}(K)<1$ hold. Let all the zeros of $K$ be in $C_{-}$. Then (1.1) is input-to-state $L^{2}$-stable.

\section{LOWER ESTIMATES FOR QUASI-POLYNOMIALS}

In this section we present some auxiliary results, which will be used in the next sections. Consider the function

$$
k(z)=z\left(1-\int_{0}^{\eta} e^{-\tau z} d \tilde{\mu}\right)+\int_{0}^{\eta} e^{-\tau z} d \mu \quad(z \in \mathbb{C}),
$$

where $\mu=\mu(\tau)$ and $\tilde{\mu}=\tilde{\mu}(\tau)$ are nondecreasing functions defined on $[0, \eta]$, and satisfying the conditions

$$
0<\operatorname{var}(\tilde{\mu})<1, \text { and } \operatorname{var}(\mu)<\infty \text {. }
$$

Put

$$
v_{1}=\frac{2 \operatorname{var}(\mu)}{1-\operatorname{var}(\tilde{\mu})} .
$$

The following two lemmas are proved in [11] (Lemmas 7 and 9).

Lemma 5. Under conditions (4.2) the equality

$$
\inf _{-\infty \leq \omega \leq \infty}|k(i \omega)|=\inf _{-v_{1} \leq \omega \leq v_{1}}|k(i \omega)|
$$

is valid. 
Lemma 6. Let the conditions (4.2),

$$
\eta v_{1}<\pi / 2 \text { and } d_{0}:=\int_{0}^{\eta} \cos \left(v_{1} \tau\right) d \mu-v_{1} \int_{0}^{\eta} \sin \left(v_{1} \tau\right) d \tilde{\mu}>0
$$

hold. Then all the zeros of $k($.$) are in C_{-}$and

$$
\inf _{-\infty \leq \omega \leq \infty}|k(i \omega)| \geq d_{0}>0
$$

For instance consider the function

$$
k_{1}(z)=z\left(1-\tilde{a} e^{-\tilde{h} z}\right)+a e^{-h z}+b
$$

with $a, b, h, \tilde{h}=$ const $\geq 0$, and $0<\tilde{a}<1$. Then

$$
v_{1}=\frac{2(a+b)}{1-\tilde{a}} .
$$

Now Lemma 6 implies

Corollary 7. Let $h v_{1}<\pi / 2, \tilde{h} v_{1}<\pi / 2$ and

$$
d_{1}:=a \cos \left(v_{1} h\right)+b-v_{1} \tilde{a} \sin \left(v_{1} \tilde{h}\right)>0 .
$$

Then all the zeros of $k_{1}($.$) are in C_{-}$and $\inf _{-\infty \leq \omega \leq \infty}|k(i \omega)| \geq d_{1}>0$.

Note that recently various interesting stability criteria for neutral type time delay linear autonomous systems were suggested, cf. $[4,13,16,18]$ but the minimum for $k_{1}(i \omega)$ was not investigated.

\section{Systems With DiscRETE DELAYS IN LINEAR PARTS}

Let $\tilde{A}=\left(\tilde{a}_{j k}\right), A=\left(a_{j k}\right)$ and $C=\left(c_{j k}\right)$ be real $n \times n$-matrices. In this section we illustrate Theorem 4 in the case of the equation

$$
\dot{y}(t)-\tilde{A} \dot{y}(t-\tilde{h})+A y(t-h)+C y(t)=[F y](t)+u(t) \quad(t \geq 0),
$$

assuming that $\|\tilde{A}\|_{n}<1$. So $K(z)=z\left(I-\tilde{A} e^{-\tilde{h} z}\right)+A e^{-h z}+C$. The entries of $K$ are

$$
k_{j k}(z)=z\left(1-\tilde{a}_{j k} e^{-\tilde{h} z}\right)+a_{j k} e^{-h z}+c_{j k}(j, k=1, \ldots, n) .
$$


As it was shown by Ostrowski [17], the inequality

$$
|\operatorname{det} A| \geq \prod_{j=1}^{n}\left(\left|a_{j j}\right|-\sum_{m=1, m \neq j}^{n}\left|a_{j m}\right|\right)
$$

is valid, provided

$$
\left|a_{j j}\right|>\sum_{m=1, m \neq j}^{n}\left|a_{j m}\right| \quad(j=1, \ldots, n)
$$

Hence,

$$
|\operatorname{det} K(z)| \geq \prod_{j=1}^{n}\left(\left|k_{j j}(z)\right|-\sum_{m=1, m \neq j}^{n}\left|k_{j m}(z)\right|\right),
$$

provided the right-hand part is positive.

Furthermore, in the case $(5.1)$ we have $\operatorname{var}(\tilde{R})=\|\tilde{A}\|_{n}, \operatorname{var}(R)=\|A\|_{n}+$ $\|C\|_{n}$

$$
v_{0}=\frac{2\left(\|A\|_{n}+\|C\|_{n}\right)}{1-\|\tilde{A}\|_{n}}
$$

So

$$
N_{2}(K(i \omega)) \leq \zeta(K) \quad\left(|\omega| \leq v_{0}\right)
$$

where $\zeta(K)=v_{0}\left(\sqrt{n}+N_{2}(\tilde{A})\right)+N_{2}(A)+N_{2}(C)$. For instance, consider the system

$$
\dot{y}_{j}(t)-\tilde{a}_{j j} \dot{y}_{j}(t-\tilde{h})+\sum_{k=1}^{n}\left(a_{j k} y_{k}(t-h)+c_{j k} y_{k}(t)\right)=\left[F_{j} y\right](t)+u(t)
$$

$(j=1, \ldots, n ; t \geq 0)$, where $F_{j} y$ are coordinates of $F y$, and suppose that

$$
\tilde{a}_{j j}, a_{j j}, c_{j j} \geq 0
$$

So $\tilde{A}=\operatorname{diag}\left(\tilde{a}_{j j}\right)$. Note that $\tilde{a}_{j j} \leq\|\tilde{A}\|_{n}<1$, put

$$
w_{j}=\frac{2\left(a_{j j}+c_{j j}\right)}{1-\tilde{a}_{j j}}
$$


and assume that

$$
w_{j} \max \{h, \tilde{h}\}<\frac{\pi}{2} \text {, and } d_{j}:=a_{j j} \cos \left(w_{j} h\right)+c_{j j}-w_{j} \tilde{a} \sin \left(w_{j} \tilde{h}\right)>0
$$

$(j=1, \ldots, n)$. Then by Corollary 7 all the zeros of $k_{j j}($.$) are in C_{-}$and

$$
\inf _{-\infty \leq \omega \leq \infty}\left|k_{j j}(i \omega)\right| \geq d_{j}>0
$$

In addition, let

$$
\rho_{j}:=d_{j}-\sum_{m=1, m \neq j}^{n}\left(\left|a_{j m}\right|+\left|c_{j m}\right|\right)>0(j=1, \ldots, n) .
$$

According to (5.3) and (5.4) we get

$$
|\operatorname{det} K(i \omega)| \geq \prod_{j=1}^{n} \rho_{j}
$$

Thus by (5.4) and Theorem 4 we arrive at the following result.

Corollary 8. Let the conditions (2.1), (5.7), (5.8) and

$$
q \zeta^{n-1}(K)<(n-1)^{(n-1) / 2} \prod_{j=1}^{n} \rho_{j}
$$

be fulfilled. Then system (5.5) is input-to-state $L^{2}$-stable.

\section{EXAMPLE}

Consider the system

$$
\dot{y}_{j}(t)-a \dot{y}_{j}(t-h)+\sum_{k=1}^{2} c_{j k} y_{k}(t)=f_{j}\left(y_{1}\left(t-h_{1}\right), y_{2}\left(t-h_{2}\right)\right)+u_{j}(t)
$$

$(j=1,2 ; t \geq 0)$, where $0<a<1, c_{j k}$ are real constants, $0 \leq h_{1}, h_{2} \leq h, f_{j}$ are scalar continuous functions defined on $\mathbb{R}^{2}$ with the property

$$
\begin{gathered}
\left|f_{j}\left(z_{1}, z_{2}\right)\right| \leq \tilde{q}_{j 1}\left|z_{1}\right|+\tilde{q}_{j 2}\left|z_{2}\right| \\
\left(\tilde{q}_{j k}=\text { const } ; z_{j} \in \mathbb{R} ; j, k=1,2\right) .
\end{gathered}
$$


Besides, by the Schwarz inequality

$$
\begin{aligned}
& \int_{0}^{\infty}\left|f_{j}\left(w_{1}\left(t-h_{1}\right), w_{2}\left(t-h_{2}\right)\right)\right|^{2} d s \\
& \leq \int_{0}^{\infty}\left(\tilde{q}_{j 1}\left|w_{1}\left(t-h_{1}\right)\right|+\tilde{q}_{j 2}\left|w_{2}\left(t-h_{1}\right)\right|\right)^{2} d t \\
& \leq\left(\tilde{q}_{j 1}^{2}+\tilde{q}_{j 2}^{2}\right)\left(\int_{0}^{\infty}\left|w_{1}\left(t-h_{1}\right)\right|^{2}+\left|w_{2}\left(t-h_{1}\right)\right|^{2} d t\right) .
\end{aligned}
$$

Thus condition (2.1) holds with $n=2$ and

$$
q^{2}=\sum_{j=1}^{2} \sum_{k=1}^{2} \tilde{q}_{j k}^{2} .
$$

Furthermore, we have $K(z)=z\left(1-a e^{-z h}\right) I+C$ with $C=\left(c_{j k}\right)$,

$$
v_{0}=2(1-a)^{-1}\|C\|_{2}
$$

and

$$
N_{2}(K(i \omega)) \leq \gamma_{K}:=v_{0} \sqrt{2}(1+a)+N_{2}(C) \quad\left(|\omega| \leq v_{0}\right)
$$

Since $\tilde{A}$ and $C$ commute, the characteristic values of $K$ are

$$
\lambda_{j}(K(z))=z-z a e^{-z h}+\lambda_{j}(C) .
$$

Suppose $\lambda_{k}(C)(k=1,2)$ are positive and put $v_{k}=\frac{2 \lambda_{k}(C)}{1-a}$. If

$$
h v_{k}<\pi / 2 \text { and } d_{k}:=\lambda_{k}(C)-v_{k} a \sin \left(h v_{k}\right)>0 \quad(k=1,2),
$$

then by Corollary 7 , the characteristic values of $K$ are in $C_{-}$, and $\left|\lambda_{k}(K(i \omega))\right| \geq$ $d_{k}(\omega \in \mathbb{R})$. So $\theta_{d}(K) \leq \frac{\gamma_{K}}{d_{1} d_{2}}$. Thanks to Theorem 4 we can assert that system (6.1) is input-to-state $L^{2}$-stable, provided the conditions (6.3) and $q \gamma_{K}<d_{1} d_{2}$ hold, where $q$ is defined by (6.2).

\section{Concluding remarks:}

In this paper we have established the explicit input-to-state stability conditions for a wide class of neutral systems. The notion of the causal mappings enables us to consider various classes of systems from the unified point of view. As the example shows, in appropriate situations we can avoid constructing the Liapunov functionals. 


\section{REFERENCES}

[1] M. Arcak and A. Teel, Input-to-state stability for a class of Lur'e systems, Automatica 38 (11) (2002), 1945-1949. doi:10.1016/S0005-1098(02)00100-0

[2] C. Corduneanu, Functional Equations with Causal Operators, Taylor and Francis, London, 2002.

[3] A. Feintuch and R. Saeks, System Theory. A Hilbert Space Approach, Ac. Press, New York, 1982.

[4] T.T. Georgiou and M.C. Smith, Graphs, causality, and stabilizability: linear, shiftinvariant systems on $L^{2}[0,8)$, Math. Control Signals Systems 6 (1993), 195-223. doi:10.1007/BF01211620

[5] M.I. Gil', Stability of Finite and Infinite Dimensional Systems, Kluwer, N.Y, 1998 doi:10.1007/978-1-4615-5575-9

[6] M.I. Gil', On bounded input-bounded output stability of nonlinear retarded systems, Robust and Nonlinear Control 10 (2000), 1337-1344. doi:10.1002/1099-1239(20001230)10:15〈1337::AID-RNC543〉3.0.CO;2-B

[7] M.I. Gil', Operator Functions and Localization of Spectra, Lecture Notes in Mathematics, Vol. 1830, Springer-Verlag, Berlin, 2003. doi:10.1007/b93845

[8] M.I. Gil', Absolute and input-to-state stabilities of nonautonomous systems with causal mappings, Dynamic Systems and Applications 18 (2009) 655-666.

[9] M.I. Gil', $L^{2}$-absolute and input-to-state stabilities of equations with nonlinear causal mappings, Internat. J. Robust Nonlinear Control 19 (2) (2009), 151-167. doi:10.1002/rnc. 1305

[10] M.I. Gil', Stability of vector functional differential equations: a survey, Quaestiones Mathematicae 35 (2012), 1-49. doi:10.2989/16073606.2012.671261

[11] M.I. Gil', Exponential stability of nonlinear neutral type systems, Archives of Control Sci 22 (2) (2012), 125-143.

[12] M.I. Gil', Estimates for fundamental solutions of neutral type functional differential equations, Int. J. Dynamical Systems and Differential Equations 4 (4) (2012), 255-273. doi:10.1504/IJDSDE.2012.049904

[13] V.L. Kharitonov, Lyapunov functionals and Lyapunov matrices for neutral type time delay systems: a single delay case, International Journal of Control 78 (2005), 783-800. doi:10.1080/00207170500164837

[14] V.B. Kolmanovskii and V.R. Nosov, Stability of Functional Differential Equations, Academic Press, London, 1986.

[15] M. Krichman, E.D. Sontag and Y. Wang, Input-output-to-state stability, SIAM J. Control Optimization 39 (6) (2000), 1874-1928. doi:10.1137/S0363012999365352

[16] J.-J. Loiseau, M. Cardelli and X. Dusser, Neutral-type time-delay systems that are not formally stable are not BIBO stabilizable, IMA J. Math. Control Inform. 19 (2002), 217-227. doi:10.1093/imamci/19.1_and_2.217 
[17] A.M. Ostrowski, Note on bounds for determinants with dominant principal diagonals, Proc. of AMS 3 (1952), 26-30.

[18] J. Partingtona and C. Bonnetb, $H^{\infty}$ and BIBO stabilization of delay systems of neutral type, Systems \& Control Letters 52 (2004), 283-288. doi:10.1016/j.sysconle.2003.09.014

[19] R. Rakkiyappan and P. Balasubramaniam, LMI conditions for global asymptotic stability results for neutral-type neural networks with distributed time delays, Appl. Math. and Comput. 204 (2008), 317-324. doi:10.1016/j.amc.2008.06.049

[20] E.D. Sontag, Mathematical Control Theory: Deterministic Finite Dimensional Systems, Springer-Verlag, New York, 1990.

Received 8 January 2012 